\title{
Fitofagia de Podisus nigrispinus em algodoeiro e plantas daninhas
}

\author{
Walter Santos Evangelista Júnior ${ }^{(1)}$, Manoel Guedes Correa Gondim Junior ${ }^{(2)}$, Jorge Braz Torres ${ }^{(2)}$ \\ e Edmilson Jacinto Marques ${ }^{(2)}$
}

\begin{abstract}
(1)Universidade Federal de Viçosa, Dep. de Biologia Animal, CEP 36571-000 Viçosa, MG. E-mail: evangelistajr@vicosa.ufv.br (2)Universidade Federal Rural de Pernambuco, Dep. de Agronomia e Fitossanidade, Av. Dom Manoel de Medeiros s/no, Dois Irmãos, CEP 52171-900 Recife, PE. E-mail: manoguedes@hotmail.com, jtorres@bugs.ent.uga.edu, emar@ufrpe.br
\end{abstract}

\begin{abstract}
Resumo - O objetivo deste trabalho foi avaliar o efeito de seis plantas daninhas e do algodoeiro no desenvolvimento, reprodução e sobrevivência do percevejo predador Podisus nigrispinus (Dallas) (Heteroptera: Pentatomidae) sob escassez parcial de presas, alimentação em intervalos de três dias, e ausência total de presas. Com escassez parcial de presas, o desenvolvimento ninfal foi maior em Ricinus communis e menor em Bidens pilosa. Viabilidade dos ínstares, peso de fêmeas, período de pré-oviposição e fecundidade foram similares entre as plantas, porém o peso de machos e longevidade de fêmeas foram reduzidos em Desmodium tortuosum e R. communis, respectivamente. Com base nos parâmetros de tabela de vida foi estimada melhor performance do predador em Amaranthus hybridus, $D$. tortuosum e $R$. communis. Ninfas submetidas à escassez total de presas viveram mais em Ageratum conyzoides, B. pilosa, D. tortuosum e Euphorbia heterophylla; porém não viveram além do terceiro ínstar. A longevidade de fêmeas do predador foi favorecida pela presença de A. conyzoides em relação a Gossypium hirsutum, vivendo em média 15,7 e 29,8 dias, respectivamente. No entanto, a disponibilidade de plantas não foi suficiente para as fêmeas atingirem maturação sexual e produção de ovos, quando submetidas à escassez total de presas.
\end{abstract}

Termos para indexação: Asopinae, controle biológico, tabela de vida, zoofitofagia.

\section{Phytophagy by Podisus nigrispinus on cotton plants and weeds}

\begin{abstract}
This work evaluated the effect of six weeds and cotton plants on predatory stinkbug Podisus nigrispinus (Dallas) (Heteroptera: Pentatomidae) development, reproduction and survival under partial prey scarcity, three days feeding intervals, and total absence of prey. Nymphal developmental periods under partial prey scarceness with three days of feeding intervals were higher on Ricinus communis and lower on Bidens pilosa. Partial prey scarcity did not affect nymphal viability, adult female weight and reproductive characteristics, but male weight and female longevity were reduced in Desmodium tortuosum and $R$. communis, respectively. Podisus nigrispinus showed better performance on Amaranthus hybridus, D. tortuosum and $R$. communis based on the life table parameters. Nymphs reared under total absence of prey lived longer on Ageratum conyzoides, B. pilosa, D. tortuosum and Euphorbia heterophylla; however, they did not live beyond the third ínstar. Longevity of P. nigrispinus females was improved depending on the plant available ranging from 15.7 days on Gossypium hirsutum to 29.8 days on A. conyzoides. The plant availability under total absence of prey, however, was not sufficient to endure female sexual maturation and egg production.
\end{abstract}

Index terms: Asopinae, biological control, life table, zoophytophagy.

\section{Introdução}

Percevejos predadores Asopinae apresentam mecanismos de adaptação no aparelho bucal e no complexo enzimático digestivo, tornando-os aptos a exercerem ocasionalmente fitofagia sem causar danos às plantas hospedeiras (Cohen, 1996), o que permite a estes predadores obtenção de umidade e, possivelmente, complemento alimentar especialmente em situações de escassez de presas (Coll \& Guershon, 2002). Tal fato tem sido objeto de estudos com o predador Podisus nigrispinus (Dallas) (Heteroptera: Pentatomidae) e os resultados indicam melhorias nas características biológicas mediante o rápido desenvolvimento ninfal, maior longevidade e fecundidade (Zanuncio et al., 1993; Oliveira et al., 2002). Coll \& Guershon (2002) sugerem que percevejos predadores podem ser classificados em zoofitófagos ou fitozoófagos, dependendo da importância da presa e da planta para o seu desenvolvimento e 
reprodução. Assim, segundo Oliveira et al. (2002), $P$. nigrispinus situa-se como zoofitófago, pois, embora receba efeito direto das plantas nas suas características biológicas, não consegue se desenvolver na ausência de presas tendo acesso somente a plantas.

A diversificação vegetal dos agroecossistemas poderá ou não beneficiar a colonização de espécies do gênero Podisus, já que ao exercerem a fitofagia, esses insetos podem ser afetados por compostos secundários presentes nas plantas (Valicente \& O'Neil, 1995; Traugott $\&$ Stamp, 1997). Por outro lado, percevejos predadores podem ser beneficiados com a presença de plantas, especialmente leguminosas, quando utilizadas em complemento à dieta (Naranjo \& Stimac, 1985; Molina-Rugama et al., 1997) ou quando a presa é de baixa qualidade nutricional (Vivan et al., 2003). Logo, a fitofagia exercida por Podisus sugere que a composição vegetal dos agroecossistemas terá importante papel na biologia de $P$. nigrispinus em períodos de escassez de presas. No entanto, a destruição dos restos culturais é prática regulamentar no manejo integrado de pragas na cultura do algodoeiro (Santos, 1999). Desta maneira, predadores e parasitóides, bem como as pragas dependem das plantas daninhas próximas aos plantios para se manterem no ambiente, o que é considerado um importante fator no estabelecimento e colonização de inimigos naturais (Altieri, 1989). Além do alimento fornecido por meio de presas, pólen, néctar e refúgio para os inimigos naturais, de modo geral a manipulação de plantas daninhas próximas à área de cultivo poderá beneficiar a manutenção e sustentação de populações de percevejos predadores pela fitofagia. O objetivo deste trabalho foi avaliar o efeito de plantas daninhas e do algodoeiro no desenvolvimento, reprodução e sobrevivência de Podisus nigrispinus sob condições de escassez parcial, alimentação em intervalos de três dias, e ausência total de presas.

\section{Material e Métodos}

\section{Seleção, obtenção e condução das plantas daninhas}

As plantas dicotiledôneas por apresentarem, geralmente, caule ereto, folhas largas, menos lignificadas, teoricamente beneficiarão a colonização de percevejos predadores mediante fitofagia. Por esta razão, plantas daninhas dicotiledôneas foram selecionadas baseandose no levantamento feito por Beltrão \& Azevedo (1994), que apresentaram as espécies de plantas daninhas mais freqüentemente associadas à cultura do algodoeiro no Nordeste do Brasil. As espécies escolhidas foram: caruru [Amaranthus hybridus L. (Amaranthaceae)], carrapicho [Desmodium tortuosum (Sw.) (Leguminoseae)], leiteiro [Euphorbia heterophylla L. (Euphorbiaceae)], mentrasto [Ageratum conyzoides L. (Compositae)], picão-preto [Bidens pilosa L. (Compositae)] e mamona [Ricinus communis L. (Euphorbiaceae)]. Essas espécies foram estudadas comparando-as com a planta cultivada do algodoeiro [Gossypium hirsutum L. r. latifolium Hutch. (Malvaceae)] cv. CNPA Precoce 1.

Plantas daninhas, na fase vegetativa usadas nos experimentos, foram obtidas de sementes colhidas de plantas presentes no Departamento de Agronomia da Universidade Federal Rural de Pernambuco (UFRPE), Recife, PE e mantidas em refrigerador a $10^{\circ} \mathrm{C}$. Sementes do algodoeiro variedade CNPA Precoce 1 foram obtidas na Embrapa Algodão, Campina Grande, PB. As plantas foram cultivadas em vasos de plástico de $5 \mathrm{~L}$, contendo mistura de solo esterilizado e húmus de minhoca na proporção de 4:1. Durante a fase experimental, foram utilizadas plantas com idade de 40 a 70 dias e para isto, os plantios foram realizados em intervalos de 20 dias.

\section{Obtenção do predador $P$. nigrispinus}

Ovos do predador utilizados nos experimentos foram oriundos da colônia mantida no Laboratório de Controle Biológico da UFRPE. O experimento foi realizado em telado a $29 \pm 6^{\circ} \mathrm{C}, 75 \pm 6 \%$ de UR e fotoperíodo natural de aproximadamente 12:12 horas. Ovos com idade inferior a 24 horas foram confinados sobre as plantas em grupos de seis, utilizando-se gaiolas de tecido de organza medindo $30 \mathrm{~cm}$ de comprimento e $15 \mathrm{~cm}$ de largura, fixadas à haste principal das plantas. Em plantas de algodoeiro, o confinamento foi realizado na terceira ou quarta folha desenvolvida do ápice da planta. Cada tratamento constou de seis repetições, com um total de 36 ovos por tratamento em delineamento experimental inteiramente casualizado. A partir do segundo ínstar foram fornecidas duas pupas de Tenebrio molitor (L.) (Coleoptera: Tenebrionidae) como presa. A presa era oferecida ao predador nas gaiolas durante 24 horas, retirada após este período e reposta em intervalos de três dias ou não era fornecida, induzindo uma situação de escassez parcial e total de alimento, respectivamente. As avaliações foram realizadas duas vezes ao dia, em intervalos de 12 horas, anotando-se a duração e a viabi- 
lidade de cada ínstar e peso de adultos no dia da emergência. Após a identificação do sexo e pesagem, fêmeas e machos foram mantidos separadamente, nos respectivos tratamentos por três dias para a maturação sexual. Em seguida, as fêmeas ( $\mathrm{n}=12$ repetições) foram acasaladas individualmente e mantidas sobre as mesmas plantas, na densidade de um casal por gaiola. Avaliou-se diariamente a longevidade e fecundidade das fêmeas. Na avaliação da viabilidade dos ovos, as posturas coletadas foram acondicionadas em placas de Petri contendo um pedaço de algodão umedecido com água até a eclosão das ninfas.

Com os resultados obtidos foram elaboradas tabelas de vida de fertilidade, determinando-se a taxa líquida de reprodução $\left(\mathrm{R}_{\mathrm{O}}\right)$, tempo médio de geração $(\mathrm{T})$, taxa intrínseca de crescimento populacional $\left(\mathrm{r}_{\mathrm{m}}\right)$ e razão finita de aumento populacional $(\lambda)$, conforme Southwood (1978).

Os dados de duração e viabilidade de ninfas, peso de adultos, longevidade de fêmeas, período de préoviposição, fecundidade, bem como os parâmetros da tabela de vida de fertilidade foram submetidos ao teste de Lavene para verificação da homogeneidade da variância. Em seguida, foram submetidos à análise de variância e as médias comparadas a 5\% de probabilidade pelo teste de Tukey. As análises foram realizadas pelo sistema computacional SANEST 4.0.

\section{Escassez total de presa}

$\mathrm{O}$ experimento foi realizado nas mesmas condições e local descritos anteriormente diferenciando apenas pela escassez total de presas. As avaliações foram realizadas duas vezes ao dia, em intervalos de 12 horas, observando-se a mudança de ínstar e a viabilidade das ninfas. $\mathrm{Na}$ avaliação da escassez total de presa na reprodução, 12 casais de $P$. nigrispinus com três dias de idade, ob- tidos da criação de laboratório com total disponibilidade de alimento, foram acasalados sobre as plantas por 72 horas; após este período os machos foram retirados para evitar canibalismo. Nesta fase avaliaram-se a produção de ovos e a longevidade das fêmeas. Tanto as ninfas como os adultos foram mantidos em condições de ausência total de presas. Os dados foram submetidos à análise de variância e as médias comparadas a 5\% de probabilidade pelo teste de Tukey. As análises foram realizadas pelo sistema computacional SANEST 4.0.

\section{Resultados e Discussão}

\section{Escassez parcial de presas}

A disponibilidade do algodoeiro e de plantas daninhas, em condições de escassez parcial de presas não afetou a duração dos ínstares de P. nigrispinus (Tabela 1), bem como a viabilidade dos ínstares e da fase ninfal (Tabela 2). No entanto, o retardo temporal cumulativo dos ínstares promoveu alteração no período ninfal, com plantas de $R$. communis prolongando a duração da fase ninfal, em relação a $B$. pilosa que proporcionou a menor duração (Tabela 1).

A disponibilidade do algodoeiro e de plantas daninhas em condições de escassez parcial de presas afetou o peso de machos recém-emergidos que variou de 32,4 a $40,5 \mathrm{mg}$, sendo mais pesados quando criados em A. hybridus, G. hirsutum e R. communis, em relação a D. tortuosum. Por outro lado, o peso de fêmeas recémemergidas não foi afetado ( $\mathrm{P}>0,05)$, e variou de 50,2 a $58,3 \mathrm{mg}$ (Tabela 3 ). O peso de adultos, especialmente, de fêmeas pode indicar o sucesso reprodutivo de percevejos do gênero Podisus (Evans, 1982). Os estudos de Evans (1982) e de Zanuncio et al. (1992) indicam que fêmeas mais pesadas apresentam maior longevidade e

Tabela 1. Duração (dias) dos ínstares e do período ninfal de Podisus nigrispinus confinados em algodoeiro e em plantas daninhas, sob condições de escassez parcial de presa, a $29 \pm 6^{\circ} \mathrm{C}, 75 \pm 6 \%$ de UR e fotófase de 12 horas, em telado ${ }^{(1)}$.

\begin{tabular}{lcccccc}
\hline Planta & \multicolumn{5}{c}{ Ínstares } & Período ninfal \\
\cline { 2 - 6 } & $\mathrm{I}$ & $\mathrm{II}$ & $\mathrm{III}$ & $\mathrm{IV}$ & $\mathrm{V}$ \\
\hline Ageratum conyzoides & $2,0 \pm 0,00 \mathrm{a}$ & $3,2 \pm 0,09 \mathrm{a}$ & $3,5 \pm 0,17 \mathrm{a}$ & $3,3 \pm 0,18 \mathrm{a}$ & $6,4 \pm 0,40 \mathrm{a}$ & $18,5 \pm 0,25 \mathrm{ab}$ \\
Amaranthus hybridus & $2,0 \pm 0,00 \mathrm{a}$ & $3,7 \pm 0,31 \mathrm{a}$ & $3,6 \pm 0,14 \mathrm{a}$ & $3,4 \pm 0,19 \mathrm{a}$ & $5,8 \pm 0,35 \mathrm{a}$ & $18,5 \pm 0,22 \mathrm{ab}$ \\
Bidens pilosa & $2,0 \pm 0,00 \mathrm{a}$ & $3,2 \pm 0,08 \mathrm{a}$ & $3,7 \pm 0,07 \mathrm{a}$ & $3,3 \pm 0,10 \mathrm{a}$ & $5,9 \pm 0,31 \mathrm{a}$ & $18,0 \pm 0,19 \mathrm{~b}$ \\
Desmodium tortuosum & $2,1 \pm 0,05 \mathrm{a}$ & $2,9 \pm 0,03 \mathrm{a}$ & $3,5 \pm 0,03 \mathrm{a}$ & $3,4 \pm 0,13 \mathrm{a}$ & $7,1 \pm 0,20 \mathrm{a}$ & $18,9 \pm 0,09 \mathrm{~b}$ \\
Euphorbia heterophylla & $2,0 \pm 0,00 \mathrm{a}$ & $3,2 \pm 0,11 \mathrm{a}$ & $3,6 \pm 0,09 \mathrm{a}$ & $3,3 \pm 0,13 \mathrm{a}$ & $6,3 \pm 0,34 \mathrm{a}$ & $18,4 \pm 0,31 \mathrm{ab}$ \\
Gossypium hirsutum & $2,0 \pm 0,00 \mathrm{a}$ & $3,4 \pm 0,37 \mathrm{a}$ & $3,6 \pm 0,18 \mathrm{a}$ & $3,4 \pm 0,19 \mathrm{a}$ & $6,6 \pm 0,37 \mathrm{a}$ & $19,0 \pm 0,48 \mathrm{ab}$ \\
Ricinus communis & $2,0 \pm 0,00 \mathrm{a}$ & $3,7 \pm 0,29 \mathrm{a}$ & $3,8 \pm 0,18 \mathrm{a}$ & $4,4 \pm 0,64 \mathrm{a}$ & $6,0 \pm 0,40 \mathrm{a}$ & $20,0 \pm 0,82 \mathrm{a}$ \\
\hline
\end{tabular}

${ }^{(1)}$ Médias \pm erro-padrão seguidas de mesma letra não diferem entre si a $5 \%$ de probabilidade pelo teste de Tukey. 
Tabela 2. Viabilidade (\%) de ínstares e da fase ninfal de Podisus nigrispinus confinados em algodoeiro e em plantas daninhas, sob condições de escassez parcial de presa, a $29 \pm 6^{\circ} \mathrm{C}, 75 \pm 6 \%$ de UR e fotófase de 12 horas, em telado ${ }^{(1)}$.

\begin{tabular}{lrrrrrr}
\hline \multirow{2}{*}{ Planta } & \multicolumn{3}{c}{ Ínstares } & Período ninfal \\
\cline { 2 - 6 } & \multicolumn{1}{c}{$\mathrm{I}$} & \multicolumn{1}{c}{ II } & \multicolumn{1}{c}{ III } & \multicolumn{1}{c}{ IV } & V \\
\hline Ageratum conyzoides & $100,0 \pm 0,00 \mathrm{a}$ & $97,2 \pm 2,78 \mathrm{a}$ & $100,0 \pm 0,00 \mathrm{a}$ & $93,9 \pm 2,78 \mathrm{a}$ & $93,3 \pm 6,67 \mathrm{a}$ & $95,8 \pm 4,17 \mathrm{a}$ \\
Amaranthus hybridus & $100,0 \pm 0,00 \mathrm{a}$ & $95,8 \pm 4,17 \mathrm{a}$ & $97,2 \pm 2,78 \mathrm{a}$ & $96,7 \pm 3,33 \mathrm{a}$ & $100,0 \pm 0,00 \mathrm{a}$ & $93,1 \pm 4,52 \mathrm{a}$ \\
Bidens pilosa & $100,0 \pm 0,00 \mathrm{a}$ & $100,0 \pm 0,00 \mathrm{a}$ & $100,0 \pm 0,00 \mathrm{a}$ & $97,2 \pm 2,78 \mathrm{a}$ & $100,0 \pm 0,00 \mathrm{a}$ & $97,2 \pm 2,78 \mathrm{a}$ \\
Desmodium tortuosum & $100,0 \pm 0,00 \mathrm{a}$ & $100,0 \pm 0,00 \mathrm{a}$ & $97,2 \pm 2,78 \mathrm{a}$ & $100,0 \pm 0,00 \mathrm{a}$ & $100,0 \pm 0,00 \mathrm{a}$ & $97,2 \pm 2,78 \mathrm{a}$ \\
Euphorbia heterophylla & $100,0 \pm 0,00 \mathrm{a}$ & $97,2 \pm 2,78 \mathrm{a}$ & $97,2 \pm 2,78 \mathrm{a}$ & $97,2 \pm 2,78 \mathrm{a}$ & $100,0 \pm 0,00 \mathrm{a}$ & $92,9 \pm 7,03 \mathrm{a}$ \\
Gossypium hirsutum & $100,0 \pm 0,00 \mathrm{a}$ & $100,0 \pm 0,00 \mathrm{a}$ & $100,0 \pm 0,00 \mathrm{a}$ & $92,5 \pm 4,17 \mathrm{a}$ & $100,0 \pm 0,00 \mathrm{a}$ & $95,8 \pm 4,17 \mathrm{a}$ \\
Ricinus communis & $100,0 \pm 0,00 \mathrm{a}$ & $91,7 \pm 5,69 \mathrm{a}$ & $88,9 \pm 8,24 \mathrm{a}$ & $100,0 \pm 0,00 \mathrm{a}$ & $85,5 \pm 8,01 \mathrm{a}$ & $75,0 \pm 12,72 \mathrm{a}$ \\
\hline
\end{tabular}

${ }^{(1)}$ Médias terro-padrão seguidas de mesma letra não diferem entre si a 5\% de probabilidade pelo teste de Tukey.

Tabela 3. Peso de adultos recém emergidos de Podisus nigrispinus confinados em algodoeiro e em plantas daninhas, sob condições de escassez parcial de presa, a $29 \pm 6^{\circ} \mathrm{C}, 75 \pm 6 \%$ de UR e fotófase de 12 horas, em telado ${ }^{(1)}$.

\begin{tabular}{llr}
\hline Planta & \multicolumn{2}{c}{ Peso (mg) } \\
\cline { 2 - 3 } & \multicolumn{1}{c}{ Macho } & Fêmea \\
\hline Ageratum conyzoides & $35,8 \pm 3,41 \mathrm{ab}$ & $57,3 \pm 4,42 \mathrm{a}$ \\
Amaranthus hybridus & $40,5 \pm 2,35 \mathrm{a}$ & $58,3 \pm 3,98 \mathrm{a}$ \\
Bidens pilosa & $37,3 \pm 1,84 \mathrm{ab}$ & $51,8 \pm 3,57 \mathrm{a}$ \\
Desmodium tortuosum & $32,4 \pm 2,83 \mathrm{~b}$ & $50,2 \pm 4,07 \mathrm{a}$ \\
Euphorbia heterophylla & $35,8 \pm 2,65 \mathrm{ab}$ & $56,0 \pm 3,85 \mathrm{a}$ \\
Gossypium hirsutum & $39,6 \pm 3,62 \mathrm{a}$ & $58,0 \pm 3,17 \mathrm{a}$ \\
Ricinus communis & $39,2 \pm 3,79 \mathrm{a}$ & $57,3 \pm 4,52 \mathrm{a}$ \\
\hline
\end{tabular}

(1) Médiasıerro-padrão seguidas de mesma letra não diferem entre si a $5 \%$ de probabilidade pelo teste de Tukey.

capacidade reprodutiva. Considerando o peso de fêmeas de $P$. nigrispinus obtidas neste trabalho, todas as plantas proporcionaram resultado similar e poderão auxiliar positivamente na colonização e manutenção deste predador no agroecossistema algodoeiro.

O período de pré-oviposição e a fecundidade não foram afetados pela disponibilidade do algodoeiro e plantas daninhas em condições de escassez parcial de presa; no entanto, a viabilidade de ovos e a longevidade apresentaram diferenças (Tabela 4). A viabilidade de ovos foi maior em $R$. communis $(72,9 \%)$, diferindo apenas de E. heterophylla $(53,9 \%)$ e G. hirsutum (48,7\%). Fêmeas de P. nigrispinus viveram de 31,4 a 47,2 dias, dependendo da planta disponível com a maior longevidade observada em $D$. tortuosum, porém sendo superior apenas a $R$. communis (Tabela 4).

Todos os parâmetros da tabela de vida de fertilidade de P. nigrispinus foram afetados pelo tipo de planta testada. A taxa líquida de reprodução $\left(\mathrm{R}_{\mathrm{o}}\right)$ foi maior para D. tortuosum, diferindo das demais plantas estudadas, enquanto $R$. communis proporcionou resultado numericamente inferior (Tabela 5). Plantas de A. hybridus e $R$. communis apresentaram menor tempo médio da geração $(\mathrm{T})$, em relação às demais plantas estudadas (Tabela 5). A taxa intrínseca de crescimento populacional $\left(\mathrm{r}_{\mathrm{m}}\right)$ e a razão finita de crescimento populacional $(\lambda)$ foram significativamente superiores em relação a A. hybridus, D. tortuosum e $R$. communis em comparação com as demais plantas estudadas (Tabela 5).

A disponibilidade de plantas na dieta de percevejos predadores, geralmente, proporciona efeitos positivos no desenvolvimento ninfal e nas características reprodutivas, variando de acordo com o tipo de planta e de presa (Naranjo \& Gibson, 1996; Vivan et al., 2003). Neste trabalho, a disponibilidade das plantas resultou em duração ninfal de P. nigrispinus de 18 a 20 dias, compatível com as variações encontradas nesta espécie em temperaturas de 27 a $32^{\circ} \mathrm{C}$ (Torres et al., 1998), embora este trabalho tenha sido realizado sob condições de escassez parcial de presas. Este fato caracteriza que as plantas não tiveram efeito negativo na duração ninfal deste predador. O tipo de planta disponível afeta o desenvolvimento de $P$. nigrispinus, podendo ser acentuado com a escassez da presa em intervalos de alimentação (Crum et al., 1998; Oliveira et al., 2002), ou pela baixa qualidade da presa (Mukerji \& LeRoux, 1969; Vivan et al., 2003). O efeito negativo de determinadas plantas sobre o desenvolvimento de Podisus advém da defesa intrínseca das plantas, mediante compostos secundários (Cohen, 1996). Valicente \& O'Neil (1995) verificaram que as solanáceas Solanum tuberosum L. e Lycopersicon esculetum Mill. apresentaram efeitos negativos à biologia de Podisus maculiventris (Say) comparados à alimentação somente em presas. Da mesma forma, Traugott \& Stamp (1997) observa- 
ram que os compostos tomatina e ácido clorogênico encontrados em plantas de tomateiro afetaram o desenvolvimento de $P$. maculiventris, tanto via dieta artificial como por meio da presa. Os resultados em relação à duração da fase ninfal com plantas de $R$. communis sugerem que esta planta provavelmente seja nutricionalmente inferior às demais espécies estudadas.

A viabilidade ninfal acima de $88 \%$ em todos os ínstares e superior a 74,9\% em toda a fase ninfal pode ser considerada alta entre as plantas estudadas, mesmo quando com resultados obtidos em condições controladas e com disponibilidade total de presas, a qual pode variar de 60\% a 99\% (Zanuncio et al., 1993; Oliveira et al., 2002; Vivan et al., 2003). Naranjo \& Stimac (1985) estudaram o efeito de plantas de soja, feijão e 10 plantas daninhas associadas à cultura da soja na biologia do percevejo predador Geocoris punctipes (Say) (Heteroptera: Geocoridae), o qual apresenta comportamento de fitofagia similar a $P$. nigrispinus e observaram que não houve influência das plantas na viabilidade ninfal. Vários estudos, com diferentes níveis de escas- sez e qualidade de presas para Podisus sugerem que este predador possui a estratégia de usar plantas como fonte de umidade para elevar a viabilidade e longevidade, reduzindo outras características biológicas como peso e reprodução (O’Neil \& Wiedenmann, 1990; Crum et al., 1998; Oliveira et al., 2002). Este fato é claramente observado em relação à longevidade de adultos confinados nas plantas de A. conyzoides, B. pilosa e D. tortuosum, que mesmo em total ausência de presa viveram de três a quatro semanas (Tabela 6).

$\mathrm{O}$ peso de adultos de $P$. nigrispinus pode indicar o sucesso reprodutivo da espécie, em especial a interação peso de fêmeas e fecundidade (Evans, 1982). Este fato foi observado em $P$. nigrispinus quando confinado nas diferentes plantas daninhas e algodoeiro, obtendo peso similar, fecundidade e período de pré-oviposição, independente do intervalo de alimentação de três dias sem alimentação (Tabela 4). Isto sugere que o uso das plantas daninhas como fonte alternativa de umidade e nutriente não causaram efeito negativo, nas condições estudadas, no peso de fêmeas e na fecundidade, o que po-

Tabela 4. Parâmetros reprodutivos e longevidade de Podisus nigrispinus confinados em algodoeiro e em plantas daninhas, sob condições de escassez parcial de presa, a $29 \pm 6^{\circ} \mathrm{C}, 75 \pm 6 \%$ de UR e fotófase de 12 horas, em telado ${ }^{(1)}$.

\begin{tabular}{lllll}
\hline Planta & \multicolumn{1}{c}{$\begin{array}{c}\text { Período } \\
\text { pré-oviposição } \\
\text { (dias) }\end{array}$} & $\begin{array}{c}\text { Fecundidade } \\
(\%)\end{array}$ & $\begin{array}{c}\text { Viabilidade } \\
\text { de ovos }(\%)\end{array}$ & $\begin{array}{c}\text { Longevidade } \\
\text { (dias) }\end{array}$ \\
\hline Ageratum conyzoides & $5,0 \pm 0,83 \mathrm{a}(2,0-10,0)$ & $309,0 \pm 29,75 \mathrm{a}(115,0-437,0)$ & $63,3 \pm 3,97 \mathrm{ab}(49,1-94,6)$ & $40,0 \pm 3,14 \mathrm{ab}(19,0-57,0)$ \\
Amaranthus hybridus & $3,8 \pm 0,38 \mathrm{a}(2,0-6,0)$ & $256,1 \pm 36,28 \mathrm{a}(64,0-412,0)$ & $65,7 \pm 6,32 \mathrm{ab}(50,0-87,7)$ & $38,8 \pm 6,16 \mathrm{ab}(15,0-71,0)$ \\
Bidens pilosa & $3,9 \pm 0,54 \mathrm{a}(2,0-6,0)$ & $280,1 \pm 30,41 \mathrm{a}(148,0-487,0)$ & $57,8 \pm 3,83 \mathrm{ab}(34,1-79,7)$ & $39,9 \pm 3,63 \mathrm{ab}(26,0-64,0)$ \\
Desmodium tortuosum & $3,8 \pm 0,52 \mathrm{a}(2,0-6,0)$ & $338,1 \pm 42,51 \mathrm{a}(121,0-512,0)$ & $67,3 \pm 3,12 \mathrm{ab}(51,5-85,0)$ & $47,2 \pm 3,26 \mathrm{a}(31,0-65,0)$ \\
Euphorbia heterophylla & $3,1 \pm 0,67 \mathrm{a}(2,0-7,0)$ & $284,5 \pm 26,30 \mathrm{a}(118,0-421,0)$ & $53,9 \pm 3,86 \mathrm{~b}(51,1-84,2)$ & $42,4 \pm 3,56 \mathrm{ab}(22,0-59,0)$ \\
Gossypium hirsutum & $4,2 \pm 0,68 \mathrm{a}(2,0-10,0)$ & $299,2 \pm 41,97 \mathrm{a}(131,0-492,0)$ & $48,7 \pm 5,36 \mathrm{~b}(32,8-84,7)$ & $37,7 \pm 4,24 \mathrm{ab}(16,0-67,0)$ \\
Ricinus communis & $3,5 \pm 0,39 \mathrm{a}(2,0-6,0)$ & $238,9 \pm 24,91 \mathrm{a}(75,0-346,0)$ & $72,9 \pm 2,93 \mathrm{a}(58,5-93,6)$ & $31,4 \pm 2,82 \mathrm{~b}(12,0-43,0)$ \\
\hline
\end{tabular}

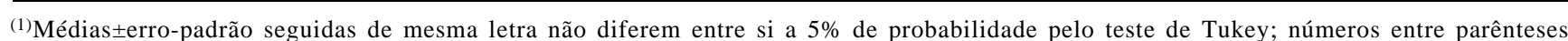
representam a maior variação entre os tratamentos.

Tabela 5. Parâmetros da tabela de vida de fertilidade de Podisus nigrispinus confinados em algodoeiro e em plantas daninhas, sob condições de escassez parcial de presa, a $29 \pm 6^{\circ} \mathrm{C}, 75 \pm 6 \%$ de UR e fotófase de 12 horas, em telado ${ }^{(1)}$.

\begin{tabular}{lcccc}
\hline Planta & $\mathrm{R}_{\mathrm{o}}$ & $\mathrm{T}$ & $\mathrm{r}_{\mathrm{m}}$ & $\ddot{\mathrm{e}}$ \\
\hline Ageratum conyzoides & $81,4 \pm 0,82 \mathrm{~b}$ & $39,0 \pm 0,13 \mathrm{ab}$ & $0,1127 \pm 0,001 \mathrm{~b}$ & $1,1188 \pm 0,001 \mathrm{~b}$ \\
Amaranthus hybridus & $69,8 \pm 1,84 \mathrm{~d}$ & $36,5 \pm 0,35 \mathrm{c}$ & $0,1161 \pm 0,001 \mathrm{a}$ & $1,1231 \pm 0,001 \mathrm{a}$ \\
Bidens pilosa & $75,3 \pm 1,09 \mathrm{c}$ & $39,8 \pm 0,25 \mathrm{a}$ & $0,1086 \pm 0,001 \mathrm{c}$ & $1,1144 \pm 0,001 \mathrm{~cd}$ \\
Desmodium tortuosum & $100,6 \pm 1,27 \mathrm{a}$ & $39,9 \pm 0,22 \mathrm{a}$ & $0,1155 \pm 0,001 \mathrm{a}$ & $1,1224 \pm 0,001 \mathrm{a}$ \\
Euphorbia heterophylla & $70,8 \pm 0,90 \mathrm{~cd}$ & $38,4 \pm 0,15 \mathrm{~b}$ & $0,1109 \pm 0,000 \mathrm{bc}$ & $1,1173 \pm 0,001 \mathrm{bc}$ \\
Gossypium hirsutum & $67,5 \pm 0,99 \mathrm{de}$ & $39,8 \pm 0,31 \mathrm{a}$ & $0,1058 \pm 0,001 \mathrm{~d}$ & $1,1116 \pm 0,001 \mathrm{~d}$ \\
Ricinus communis & $64,2 \pm 0,59 \mathrm{e}$ & $35,5 \pm 0,12 \mathrm{c}$ & $0,1172 \pm 0,000 \mathrm{a}$ & $1,1242 \pm 0,001 \mathrm{a}$ \\
\hline
\end{tabular}

${ }^{(1)}$ Médias \pm erro-padrão seguidas de mesma letra não diferem entre si a $5 \%$ de probabilidade pelo teste de Tukey; $\mathrm{R}_{\mathrm{o}}$ : taxa líquida de reprodução (descendente fêmea/fêmea); T: tempo médio de geração (dias); $\mathrm{r}_{\mathrm{m}}$ : taxa intrínsica de crescimento populacional; $\lambda$ : taxa finita de crescimento populacional (fêmeas adicionadas à população/dia). 
derá ser benéfico na manutenção do predador nos agroecossistemas quando tendo acesso a estas plantas.

Em geral, a disponibilidade de plantas apresenta incremento na produção de ovos em P. nigrispinus, quando submetidas à escassez, ou não, de presas (MolinaRugama et al., 1997; Lemos et al., 2001; Oliveira et al., 2002). A fecundidade média das fêmeas variou de 238,9 a 309,0 ovos, que embora submetidas a intervalos de três dias de alimentação e nas condições do presente trabalho, encontra-se dentro do padrão médio em relação a esta espécie em diferentes condições, desde variações na disponibilidade de presa, temperatura e plantas, que segundo Zanuncio et al. (1992) e Oliveira et al. (2002) pode ser de 140 a 609,9 ovos por fêmea quando alimentados com T. molitor. Da mesma forma, a variação na viabilidade de ovos de $P$. nigrispinus é observada quando não ocorre o controle do número e frequência de cópulas (Torres et al., 1997). A utilização de apenas um acasalamento na fase inicial de vida de fêmeas de $P$. nigrispinus pode explicar a variação e a baixa viabilidade de ovos encontrada, quando comparada ao resultado obtido por Oliveira et al. (2002), que foi acima de $70 \%$ em relação a este predador mantido em plantas de algodoeiro e tomateiro.

Os resultados da tabela de vida de fertilidade de $P$. nigrispinus indicam que a planta daninha $D$. tortuosum proporcionou melhor taxa líquida de reprodução (Tabela 5) e longevidade (Tabela 4), o que sugere maior produção de descendentes por geração, e constitui-se num parâmetro para sustentar a população desse predador no agroecossistema. Isto pode ser justificado por se tratar de uma leguminosa, sendo a família de plantas preferidas por pentatomídeos fitófagos e também por contribuírem no desenvolvimento e reprodução dos percevejos predadores (Naranjo \& Stimac, 1985; Coll, 1996).

\section{Escassez total de presa}

Ninfas de primeiro ínstar mantidas em $D$. tortuosum e E. heterophylla apresentaram viabilidade de $96,7 \%$ e $97,2 \%$, respectivamente, enquanto as demais plantas proporcionaram $100 \%$ de viabilidade (Figura 1), este resultado pode ser justificado, pelo fato que neste estádio, as ninfas não se alimentam de presa, necessitando apenas de umidade. A viabilidade de ninfas no segundo ínstar foi de $17,8 \%, 12,8 \%, 5,6 \%, 4,2 \%$ e $2,8 \%$, para G. hirsutum, B. pilosa, A. conyzoides, D. tortuosum e E. heterophylla, respectivamente, enquanto A. hybridus e $R$. communis não possibilitaram a passa- gem das ninfas para o terceiro ínstar. Ninfas de P. nigrispinus mantidas em plantas de A. conyzoides, B. pilosa, D. tortuosum e E. heterophylla sobreviveram, em média, 26,0, 22,9, 21,0 e 25,9 dias, respectivamente, sendo significativamente superior às demais plantas (Tabela 6). A baixa viabilidade no segundo ínstar pode ser justificada pelo fato que neste ínstar inicia-se a alimentação em presa. Como P. nigrispinus é considerado um percevejo zoofitófago, necessita de material animal em sua dieta, porém a sobrevivência acima de 20 dias nas plantas daninhas A. conyzoides, B. pilosa, $D$. tortuosum e E. heterophylla, permitirá a busca pela presa, conseqüentemente, possibilitará a redução da mortalidade.

Nas fêmeas, a escassez total de presa inviabilizou a

Tabela 6. Efeito de algodoeiro e de plantas daninhas na sobrevivência de ninfas e longevidade de fêmeas de Podisus nigrispinus confinadas em algodoeiro e em plantas daninhas, sob condições de escassez total de presa, a $29 \pm 6^{\circ} \mathrm{C}, 75 \pm 6 \%$ de UR e fotófase de 12 horas, em telado ${ }^{(1)}$.

\begin{tabular}{lcc}
\hline Planta & $\begin{array}{c}\text { Sobrevivência } \\
\text { (dias) }\end{array}$ & $\begin{array}{c}\text { Longevidade } \\
\text { (dias) }\end{array}$ \\
\hline Ageratum conyzoides & $26,0 \pm 1,70 \mathrm{a}$ & $29,8 \pm 1,47 \mathrm{a}$ \\
Amaranthus hybridus & $11,4 \pm 0,22 \mathrm{~b}$ & $21,1 \pm 1,49 \mathrm{bc}$ \\
Bidens pilosa & $22,9 \pm 1,72 \mathrm{a}$ & $27,3 \pm 1,45 \mathrm{ab}$ \\
Desmodium tortuosum & $21,0 \pm 1,29 \mathrm{a}$ & $25,4 \pm 1,16 \mathrm{ab}$ \\
Euphorbia heterophylla & $25,9 \pm 1,97 \mathrm{a}$ & $22,7 \pm 2,11 \mathrm{ab}$ \\
Gossypium hirsutum & $12,8 \pm 0,49 \mathrm{~b}$ & $16,3 \pm 0,85 \mathrm{c}$ \\
Ricinus communis & $12,9 \pm 0,75 \mathrm{~b}$ & $20,6 \pm 1,85 \mathrm{bc}$ \\
\hline
\end{tabular}

${ }^{(1)}$ Médias \pm erro-padrão seguidas de mesma letra não diferem entre si a $5 \%$ de probabilidade pelo teste de Tukey.

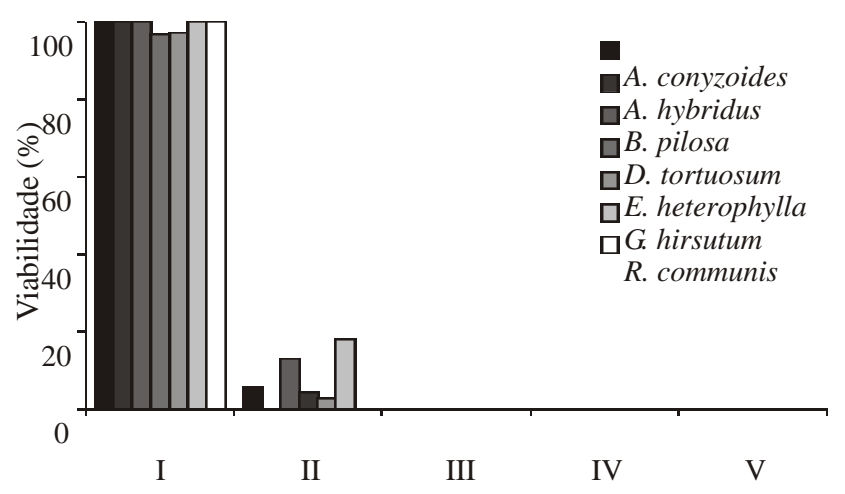

Figura 1. Viabilidade de ninfas de Podisus nigrispinus confinadas em algodoeiro e em plantas daninhas, sob condições de escassez total de presa, a $29 \pm 6^{\circ} \mathrm{C}, 75 \pm 6 \%$ de UR e fotófase de 12 horas, em telado. 
produção de ovos em $P$. nigrispinus, pois em todos os tratamentos não foi observado oviposição. Contudo, a longevidade das fêmeas foi variável de acordo com a planta. Fêmeas mantidas em A. conyzoides, B. pilosa, D. tortuosum e E. heterophylla viveram de 22,7 a 29,8 dias, sendo superior à longevidade das fêmeas mantidas em G. hirsutum (15,7 dias), enquanto fêmeas mantidas em A. hybridus e $R$. communis apresentaram longevidade intermediárias, diferindo apenas de A. conyzoides (Tabela 6 ).

A manipulação da vegetação nativa dentro ou próximo ao agroecossistema pode ajudar a reter e sustentar populações de percevejos predadores no campo por meio da fitofagia (Coll, 1995; Coll \& Guershon, 2002). Os resultados confirmam esta hipótese, pois as plantas A. conyzoides, B. pilosa, D. tortuosum e $E$. heterophylla, proporcionaram sobrevivência ninfal de $P$. nigrispinus por um período médio de 21 a 26 dias, quando confinados na ausência total de presas, enquanto ninfas mantidas em A. hybridus, G. hirsutum e R. communis viveram, em média, de 11,4 a 12,9 dias. Naranjo \& Stimac (1985) observaram que ninfas de segundo ínstar de G. punctipes sobreviveram 10 dias, em média, confinadas sobre a planta daninha Heterotheca subaxillaris (Lam.) (Compositae) e apenas 5,5 dias em plantas de soja [Glycine max (L.) Merr. (Leguminosae)]. Os resultados do presente trabalho sugerem que além dos benefícios que as plantas daninhas ou cultivadas possuem em relação aos percevejos predadores, o hábito fitofágico pode, também, ser afetado em razão da ausência ou presença de presas.

Fêmeas de $P$. nigrispinus mantidas em algodoeiro e plantas daninhas não ovipositaram na ausência total de presa. De Clercq \& Degheele (1992) mencionaram que na ausência do alimento, $P$. nigrispinus (=P. sagitta) mantém a sobrevivência, reduzindo a capacidade reprodutiva e acumulando energia na espera de melhores condições para a reprodução. Desta forma, as plantas testadas proporcionaram longevidade média acima de 15 dias em total ausência de presas. Os resultados indicam que plantas de A. conyzoides, B. pilosa e $D$. tortuosum, quando presentes no ambiente, podem manter fêmeas adultas de $P$. nigrispinus por mais de 25 dias em total ausência de presas (Tabela 6). Valicente \& O'Neil (1993) observaram que P. maculiventris sem acesso à presa e com disponibilidade das plantas de batata e tomate viveram em média 31,9 e 28,8 dias, respectivamente, ao passo que, na ausência das plantas, viveram em média 9,1 dias. Com base nos resultados de sobrevivência ninfal e longevidade de adultos de $P$. nigrispinus confinados nas plantas estudadas, podese afirmar que plantas de A. conyzoides, B. pilosa e D. tortuosum são favoráveis à manutenção de populações deste predador durante a escassez de presas.

Este trabalho apresenta o primeiro relato da interação entre plantas daninhas e o predador P. nigrispinus em situação de escassez parcial e total de presas. Com base nos resultados pode-se afirmar que as espécies $A$. conyzoides, B. pilosa e D. tortuosum, quando presentes no ambiente e utilizadas pelo predador P. nigrispinus mediante fitofagia, poderão favorecer a colonização e o estabelecimento do predador no agroecossistema algodoeiro.

\section{Conclusões}

1. As plantas Ageratum conyzoides, Bidens pilosa e Desmodium tortuosum favorecem o desenvolvimento e reprodução do predador Podisus nigrispinus comparado ao algodoeiro Gossypium hirsutum em situação de escassez de presa.

2. Sob escassez total de presas, ninfas de $P$. nigrispinus conseguem atingir o terceiro ínstar, porém com baixa viabilidade, destacando as plantas B. pilosa e G. hirsutum como os hospedeiros mais favoráveis.

3. Fêmeas de P. nigrispinus não são capazes de obterem maturação sexual e produção de ovos apenas com disponibilidade de plantas.

\section{Agradecimentos}

À Capes pelo apoio financeiro; ao Prof. Eduardo Beserra (UEPB) pelas sugestões apresentadas.

\section{Referências}

ALTIERI, M.A. Agroecologia: as bases científicas da agricultura alternativa. Rio de Janeiro: Projeto de Tecnologias Alternativas da Federação de Órgãos para Assistência Social e Educacional, 1989. 237p.

BELTRÃO, N.E.M.; AZEVEDO, D.M.P. de. Controle de plantas daninhas na cultura do algodoeiro. Campina Grande: EmbrapaCNPA, 1994. 154p.

COHEN, A.C. Plant feeding by predatory Heteroptera: evolutionary and adaptational aspects of trophic switching. In: WIEDENMANN, R.N.; ALOMAR, O. (Ed.). Zoophytophagous Heteroptera: implications for life history and integrated pest management. Lanham: Entomological Society of America, 1996. p.1-17.

COLL, M. Feeding and living on plants in predatory Heteroptera. In: COLL, M.; RUBERSON, J.R. (Ed.). Predatory Heteroptera in 
agroecosystems: their ecology and use in biological control. Lanham: Entomological Society of America, 1995. 233p.

COLL, M. Feeding and ovipositing on plants by an omnivorous insect predator. Oecologia, v.105, p.214-220, 1996.

COLL, M.; GUERSHON, M. Omnivory in terrestrial arthrods: mixing plant and prey diet. Annual Review of Entomology, v.47, p.267-297, 2002.

CRUM, D.A.; WEISER, L.A.; STAMP, N.E. Effects of prey scarcity and plant material as a dietary supplement on an insect predator. Oikos, v.81, p.549-557, 1998.

DE CLERCQ, P.; DEGHEELE, D. Influence of feeding interval on reproduction and longevity of Podisus sagitta (Het.: Pentatomidae). Entomophaga, v.37, p.583-590, 1992.

EVANS, E.W. Consequence of body size for fecundity in the predatory stinkbug, Podisus maculiventris (Hemiptera: Pentatomidae). Annals of the Entomological Society of America, v.75, p.418420, 1982

LEMOS, W.P.; MEDEIROS, R.S.; RAMALHO, F.S.; ZANUNCIO, J.C. Effects of plant feeding on the development, survival and reproduction of Podisus nigrispinus (Dallas) (Heteroptera: Pentatomidae). International Journal of Pest Management, v.47, p.89-93, 2001.

MOLINA-RUGAMA, A.J.; ZANUNCIO, J.C.; TORRES, J.B. Longevidad y fecundidad de Podisus nigrispinus (Heteroptera: Pentatomidae) alimentado con Musca domestica (Diptera: Muscidae) y fríjol. Revista de Biología Tropical, v.45, p.1125-1130, 1997.

MUKERJI, M.K.; LEROUX, E.J. A quantitative study of food consumption and growth of Podisus maculiventris (Hemiptera: Pentatomidae). Canadian Entomologist, v.101, p.387-403, 1969.

NARANJO, S.E.; GIBSON, R.L. Phytophagy in predaceous heteroptera: effects on life history and population dynamics, 57-93. In: WIEDENMANN, R.N.; ALOMAR, O. (Ed.). Zoophytophagous Heteroptera: implications for life history and integrated pest management. Lanham: Entomological Society of America, 1996. 202p.

NARANJO, S.E.; STIMAC, J.L. Development, survival and reproduction of Geocoris punctipes (Hemiptera:Lygaeidae): effects of plant feeding on soybean and associated weeds. Environmental Entomology, v.14, p.523-530, 1985.

OLIVEIRA, J.E.M.; TORRES, J.B.; MOREIRA, A.F.C.; BARROS, R. Efeito das plantas do algodoeiro e do tomateiro, como complemento alimentar, no desenvolvimento e na reprodução do predador Podisus nigrispinus (Dallas)(Heteroptera:Pentatomidae). Neotropical Entomology, v.31, p.101-108, 2002.
O'NEIL, R.J.; WIEDENMANN, R.N. Body weight of Podisus maculiventris (Say) under various feeding regimens. Canadian Entomologist, v.122, p.285-294, 1990.

SANTOS, W.J. dos. Monitoramento e controle das pragas do algodoeiro. In: CIA, E.; FREIRE, E.C.; SANTOS, W.J. dos (Ed.). Cultura do algodoeiro. Piracicaba: Potafos, 1999. p.133-179.

SOUTHWOOD, T.R.E. Ecological methods with particular reference to the study of insect populations. $2^{\text {nd }} e d$. London: Chapman \& Hall, 1978. 524p.

TORRES, J.B.; ZANUNCIO, J.C.; OLIVEIRA, M.C. Mating frequency and its effect on female reproductive output in the stinkbug predator Podisus nigrispinus (Heteroptera: Pentatomidae). Medical Faculteit Landbouwwetenschappen Rijksuniversiteit Gent, v.62, p.491-498, 1997.

TORRES, J.B.; ZANUNCIO, J.C.; OLIVEIRA, H.N. Nymphal development and adult reproduction of the stinkbug predator Podisus nigrispinus (Het.: Pentatomidae) under fluctuating temperatures. Journal of Applied Entomology, v.122, p.509-514, 1998.

TRAUGOTT, M.S.; STAMP, N.E. Effects of chlorogenic acid and tomatine-fed caterpillars on performance of an insect predator. Oecologia, v.109, p.265-272, 1997.

VALICENTE, F.H.; O'NEIL, R.J. Effect of host plants and feeding regimes on selected life history characteristics of Podisus maculiventris (Say) (Heteroptera: Pentatomidae). Biological Control, v.5, p.449-461, 1995.

VALICENTE, F.H.; O'NEIL, R.J. Effects of two host plants on selected life history characteristics of Podisus maculiventris (Say) (Heteroptera: Pentatomidae) - 1: without access to prey. Anais da Sociedade Entomológica do Brasil, v.22, p.513-519, 1993.

VIVAN, L.M.; TORRES, J.B.; VEIGA, A.F.S.L. Development and reproduction of a predatory stinkbug, Podisus nigrispinus in relation to two different prey types and environmental conditions. BioControl, v.48, p.155-168, 2003.

ZANUNCIO, J.C.; BRAGANÇA, M.A.L.; DIAZ, J.L.S.; SARTÓRIO, R.C. Avaliação dos parâmetros de fecundidade de Podisus connexivus (Hemiptera: Pentatomidae) de diferentes pesos. Revista Ceres, v.39, p.591-596, 1992.

ZANUNCIO, J.C.; FERREIRA, A.T.; ZANUNCIO, T.V.; GARCIA, J.F. Influence of feeding on Eucalyptus urophylla seedlings on the development of the predatory bug Podisus connexivus (Hemiptera: Pentatomidae). Medical Faculteit Landbouwwetenschappen Rijksuniversiteit Gent, v.58, p.469$475,1993$.

Recebido em 25 de julho de 2003 e aprovado em 6 de fevereiro de 2004 